\title{
The Fourier Transform Microwave/Millimeter-wave Spectrum of YOH and YOD $\left(\tilde{X}^{1} \Sigma^{+}\right)$
}

\author{
D. T. Halfen, J. P. Keogh, and L. M. Ziurys
}

\author{
Department of Chemistry, \\ Department of Astronomy, \\ Arizona Radio Observatory, \\ and Steward Observatory, \\ University of Arizona, \\ 933 N. Cherry Avenue, \\ Tucson, AZ 85721
}




\begin{abstract}
The rotational spectrum of $\mathrm{YOH}\left(\tilde{X}^{1} \Sigma^{+}\right)$and its deuterium analog YOD have been measured using Fourier transform microwave/millimeter-wave (FTMmmW) techniques. This work is the first pure rotational study of this molecule. YOH and YOD were created in the gas phase in a supersonic expansion from a mixture of yttrium vapor, generated by laser-ablation, and $\mathrm{H}_{2} \mathrm{O}$, diluted in argon. Three transitions were measured $\mathrm{YOH}$ and two for YOD in the frequency range $15-52 \mathrm{GHz}$. These data were analyzed using a ${ }^{1} \Sigma$ Hamiltonian, and rotational and centrifugal distortion constants were refined. Based on the rotational constants, the $r_{0}$ bond lengths of $\mathrm{YOH}$ were established to be $r(\mathrm{Y}-\mathrm{O})=1.949(1) \AA$ and $r(\mathrm{O}-\mathrm{H})=0.921(1) \AA$, in agreement with past optical work. The $\mathrm{Y}-\mathrm{O}$ bond in $\mathrm{YOH}$ is longer than in $\mathrm{YO}$, which has $r(\mathrm{Y}-$ $\mathrm{O})=1.790(1) \AA$. This difference suggests the presence of a single $\mathrm{Y}-\mathrm{O}$ bond in the hydroxide and a double $\mathrm{Y}=\mathrm{O}$ bond in the oxide.
\end{abstract}

Keywords: Rotational spectroscopy, FTMmmW spectroscopy, Yttrium hydroxide (YOH), Laser ablation 


\section{Introduction}

Metal hydroxide species are common in many aspects of chemistry, including bioinorganic, surface science, and even astrochemistry. For example, both $\mathrm{ZnOH}$ and $\mathrm{MnOH}$ are important functionalities in various metalloenzymes, such as carbonic anhydrase and arginase [1]. The catalytic site of such enzymes is based on a $\mathrm{Zn}$ or Mn metal center, with a water molecule or the $\mathrm{OH}$ group as one of the ligands, depending on the $\mathrm{pH}$ environment. The geometry of the M-O-H unit, a strong nucleophile, plays a critical role in enzyme function [1]. Metal hydroxides such as $\mathrm{ZnOH}$ also form at reactive sites in zeolites, providing an acidic environment for alkane activation and dehydrogenation reactions $[2,3]$. These species are created on certain metal surfaces during corrosion processes, as well [4]. One metal-containing hydroxide species has even been detected in interstellar space: AlOH. The molecule was observed in the oxygen-rich circumstellar envelope of the red supergiant star VY CMa, where it is found to be a major gas-phase molecular carrier of aluminum [5].

High-resolution spectroscopy of alkali and alkaline-earth hydroxide species has been carried out for several decades, beginning with the work of Lide and collaborators [6-18]. More recently, investigations of aluminum and indium hydroxides have been performed, providing additional insight $[19,20]$. These species have all proved to be linear, with the exception of the indium compound, which is bent with an angle of $132^{\circ}$. Some quasilinear behavior is exhibited by $\mathrm{MgOH}$ and $\mathrm{LiOH}[6,21]$, as well, which both appear to have very low energy bending potentials. However, very few transition metal hydroxides have been investigated by gas-phase spectroscopic methods. Only $\mathrm{CuOH}, \mathrm{ZnOH}$, and $\mathrm{AgOH}$ have thus far been studied, mainly by rotational spectroscopy [22-24], and $\mathrm{YOH}$ has been examined using laser induced fluorescence 
(LIF) techniques [25]. In contrast to the majority of other hydroxides, the molecules with the late transition metals have been found to be bent, although $\mathrm{YOH}$ is linear.

The evolution from the linear to the bent geometry is thought to indicate the increasing contribution of covalent as opposed to ionic bonding in these species. Bond angles of transition metal species have been found to lie in the range $107-114^{\circ}$ [e.g. 23,24 ], very close to that of water at $104.5^{\circ}$ [26]. Quasilinear $\mathrm{LiOH}$ and $\mathrm{MgOH}$ are thought the intermediate cases: on average linear but exhibiting large amplitude bending motions [21].

Yttrium hydroxide is the only transition metal hydroxide studied thus far that is linear. This property was established by Adam et al. in 1999 , who measured the $\widetilde{B}^{1} \Pi_{r}-\tilde{X}^{1} \Sigma^{+}$and $\widetilde{C}^{1} \Sigma^{+}$ $-\tilde{X}^{1} \Sigma^{+}$transitions of YOH and YOD using LIF spectroscopy in the $500-626 \mathrm{~nm}$ region, in conjunction with a laser ablation source [25]. These authors established the ground state to be ${ }^{1} \Sigma^{+}$and determined rotational constants and vibrational frequencies for the various electronic states [25]; they also found no evidence of quasilinear behavior, analogous to the rubidium and strontium analogs. YOH was also observed in an argon matrix using Fourier transform infrared (FTIR) methods by Zhang et al. [27], who recorded the Y-O stretching frequency of this species. Zhang et al. also used DFT methods (B3LYP) to calculate the structure of YOH, which confirmed the linear geometry [27]. However, both their experimental work and potential energy surface (PES) calculations suggested that the $\mathrm{HYOH}$ species was also a stable form. Aside from these two investigations, there have been no other spectroscopic studies of this molecule.

Here we report the first pure rotational measurements of $\mathrm{YOH}$ and its deuterium isotopologue. These data were obtained using Fourier transform microwave/millimeter-wave (FTMmmW) methods, coupled with a laser ablation source. These new measurements have 
improved the previous rotational constants by factors of 10-100, and confirmed the linear structure. In this paper we present our results and analysis, and comment on the bonding in this species.

\section{Experimental}

The pure rotational spectra of $\mathrm{YOH}$ and YOD were measured using the Fourier transform microwave/millimeter-wave (FTMmmW) spectrometer in the Ziurys group. This machine is a Balle-Flygare type narrow-band instrument and operates from $4-90 \mathrm{GHz}[28,29]$. The system consists of a vacuum chamber, evacuated to $10^{-8}$ Torr with a cryopump, with two spherical mirrors positioned in a near confocal arrangement, forming a Fabry-Perot cavity. Two different sets of mirrors are used to cover the full range of the instrument. From $4-40 \mathrm{GHz}$, antennas, imbedded in the mirrors, are used for injection and detection of the radiation. In the $40-90$ GHz range, waveguide is employed to couple the radiation into and out of the cell. A supersonic jet expansion introduces the gas sample into the chamber at a $40^{\circ}$ offset from the cavity axis. Low noise amplifiers detect the radiation from the cavity in the time domain, the free induction decay (FID). The signals are recorded by a computer, and a Fourier transform of the FID produces a spectrum. Because of the beam orientation to the cavity axis, every measured transition has a Doppler doublet structure, each with a FWHM of about $5 \mathrm{kHz}$.

YOH was created in a laser ablation source from yttrium vapor and water. A Nd:YAG laser at $532 \mathrm{~nm}$ was used to ablate the metal from a rod, which was then reacted with a dilute mixture of water vapor in argon. The mixture was created by flowing argon (40 psi at $40 \mathrm{sccm}$ ) through a U-tube filled with a small amount of liquid water. For YOD, $\mathrm{D}_{2} \mathrm{O}$ was used instead of $\mathrm{H}_{2} \mathrm{O}$. Unlike our other experiments [30], use of a DC discharge after the ablation source 
(DALAS) was not necessary to generate either YOH or YOD. Use of the discharge in fact resulted in loss of the signals.

\section{Results and Analysis}

The initial search for $\mathrm{YOH}$ was based on the previous constants of Adam et al. [25]. The $J=2 \rightarrow 1$ transition near $34 \mathrm{GHz}$ was initially sought. A line was found about $3.2 \mathrm{MHz}$ from the prediction in the course of a $6 \mathrm{MHz}$ wide survey, with consecutive scans spaced by $250 \mathrm{kHz}$. This feature was found to be due to both yttrium and water. The $J=1 \rightarrow 0$ transition was then located, appearing about $1.6 \mathrm{MHz}$ away from the calculated frequency. Measurement of the $J=$ $3 \rightarrow 2$ line of $\mathrm{YOH}$ near $52 \mathrm{GHz}$ confirmed the identification.

The spectrum of YOD was more difficult to observe, even with $\mathrm{D}_{2} \mathrm{O}$ as the precursor. A $15 \mathrm{MHz}$ range near the prediction of the $J=2 \rightarrow 1$ transition was repeatedly scanned, with consecutive scans spaced by $250 \mathrm{kHz}$, as well as a $10 \mathrm{MHz}$ region near the $J=1 \rightarrow 0$ line. With significant signal-averaging, the $J=1 \rightarrow 0$ transition was discovered about $2 \mathrm{MHz}$ from the predicted frequency, followed by the $J=2 \rightarrow 1$ line. The YOD measurements needed 10 times as many pulse averages as for $\mathrm{YOH}$ to achieve a comparable signal-to-noise ratio at the same transition $J+1 \rightarrow J$

Spectra of YOH and YOD are presented in Figures 1 and 2. In Figure 1, the $J=1 \rightarrow 0,2$ $\rightarrow 1$, and $3 \rightarrow 2$ transitions of $\mathrm{YOH}$ near $17.4,34.8$, and $52.2 \mathrm{GHz}$ are shown. The Doppler doublets are indicated by the brackets above each feature. The YOH lines appear as single features, and were acquired in 500, 500, and 25,000 nozzle pulses for the $J=1 \rightarrow 0,2 \rightarrow 1$, and $3 \rightarrow 2$ transitions, respectively. Figure 2 displays the $J=1 \rightarrow 0$ and $2 \rightarrow 1$ transitions of YOD near 15.6 and $31.3 \mathrm{GHz}$. Again, each Doppler doublet is specified by the bracket above the data. No deuterium quadrupole hyperfine splittings were found in the observed YOD lines. These 
spectra were obtained by averaging 5,000 nozzle pulses each. The transition frequencies measured for $\mathrm{YOH}$ and $\mathrm{YOD}$ are listed in Table 1.

The spectra were analyzed using a ${ }^{1} \Sigma$ Hamiltonian, including rotation and centrifugal distortion interactions:

$$
\hat{\mathrm{H}}_{\mathrm{eff}}=\hat{\mathrm{H}}_{\mathrm{rot}}+\hat{\mathrm{H}}_{\mathrm{cd}}
$$

The constants determined in the fit are listed in Table 2, along with those from Adam et al. [25]. The rms of the fits are $<1 \mathrm{kHz}$ for both YOH and YOD. As can be seen in the table, the parameters $B$ and $D$ are one to two orders of magnitude more precise than those from the previous work. While both sets of constants mostly agree within the errors, the centrifugal distortion parameter is about a factor of two smaller for YOD in the analysis presented here relative to the LIF measurements: $3.9(1.4) \mathrm{kHz}$ vs. $8.2(1.7) \mathrm{kHz}$. The centrifugal distortion constant should scale approximately as $1 / \mu^{2}$, where $\mu$ is the reduced mass. Using $D=6.36(36)$ $\mathrm{kHz}$ derived for $\mathrm{YOH}$, that for YOD should be roughly $5.2 \mathrm{kHz}$ - within the uncertainty of the value determined here.

\section{Discussion}

From the rotational constants of yttrium hydroxide and its deuterium isotopologue, an $r_{0}$ structure was established for $\mathrm{YOH}$ using the non-linear fitting routine STRFIT [31]. The bond lengths were determined to be $r(\mathrm{Y}-\mathrm{O})=1.949(1) \AA$, and $r(\mathrm{O}-\mathrm{H})=0.921(1) \AA$, see Table 3 . Also listed in Table 3 are the previous experimental structure from Adam et al. and the DFT theoretical $r_{e}$ geometry from Zhang et al. [25,27]. The pure rotational values are in excellent agreement with those derived from the LIF data, and reasonably match the DFT values, given that these are for an equilibrium structure. 
Table 3 also lists the $\mathrm{Y}-\mathrm{O}$ bond length for the YO radical, $r_{0}(\mathrm{Y}-\mathrm{O})=1.790(1) \AA$ [32]. This value is significantly shorter $(0.159 \AA)$ than that found for YOH. This result suggests that there is a single $\mathrm{Y}-\mathrm{O}$ bond in $\mathrm{YOH}$ and double $\mathrm{Y}=\mathrm{O}$ bond in $\mathrm{YO}$.

In addition, Table 3 presents the structures of other metal-containing hydroxide species $[9,17,21,23,24]$. From the table, the $\mathrm{O}-\mathrm{H}$ bond length in $\mathrm{YOH}$ is in close agreement with the linear species $\mathrm{RbOH}$ and $\mathrm{SrOH}$. It does not appear anomalously short, as found for quasilinear $\mathrm{MgOH}$, which has $r(\mathrm{O}-\mathrm{H})=0.871 \AA[21]$. The shorter bond length in the magnesium compound is thought to arise from a low energy bending potential, which enables the hydrogen atom to readily move off the linear molecular axis. The average projected $\mathrm{O}-\mathrm{H}$ bond length is thus decreased. The bent hydroxide species $\mathrm{CuOH}, \mathrm{ZnOH}$, and $\mathrm{AgOH}$, in contrast, have longer $\mathrm{O}-\mathrm{H}$ bond distances by about $0.04 \AA$. Therefore, the $\mathrm{OH}$ bond appears to be slightly destabilized in the bent structures as compared to the linear species.

Linear MOH species are thought to be more ionic than their bent counterparts $[21,24]$. The degree of covalent interactions thus appears to increase in the later part of the transition metal series. At some point in the $3 d$ and $4 d$ metals, a shift from the linear to the bent hydroxide geometry occurs. Theoretical calculations for the $3 d$ elements predict $\mathrm{ScOH}$ to be linear, while those from titanium onward are bent [33]. Similar computations for the $4 d$ metals do not currently exist, except it is clear experimentally that the change occurs between YOH and $\mathrm{AgOH}$. Spectroscopic studies of more transition metal hydroxides are clearly needed to understand their structure and bonding.

This research was supported by NSF Grant CHE 10-57924. 


\section{References}

[1] Yu.G. Kolyagin, V.V. Ordomsky, Y.Z. Khimyak, A.I. Rebrov, F. Fajula, I.I. Ivanova, J. Catal. 238 (2006) 122.

[2] S. Valange, B. Onida, F. Geobaldo, E. Garrone, Z. Gabelica, Stud. Surf. Sci. Catal. 142 (2002) 215.

[3] P. A. Theil, T. E. Madey, Surf. Sci. Rep. 7 (1987) 211.

[4] D. W. Christianson, J. D. Cox, Ann. Rev. Biochem. 68 (1999) 33.

[5] E. D. Tenenbaum, L. M. Ziurys, Astrophys. J. 712 (2010) L93.

[6] K.J. Higgins, S.M. Freund, W. Klemperer, A.J. Apponi, L.M. Ziurys, J. Chem. Phys. 121 (2004) 11715.

[7] Y. Kawashima, R.D. Suenram, E. Hirota, J. Mol. Spectrosc. 175 (1996) 99.

[8] E.F. Pearson, M.B. Trueblood, J. Chem. Phys. 58 (1973) 826.

[9] C. Matsumura, D.R. Lide, Jr., J. Chem. Phys. 50 (1969) 71.

[10] D.R. Lide, Jr., R.L. Kuczkowski, J. Chem. Phys. 46 (1967) 4768.

[11] K.J. Mascaritolo, J.M. Merritt, M.C. Heaven, J. Phys. Chem. A 117 (2013) 13654.

[12] M. J. Dick, P. M. Sheridan, J.-G. Wang, S. Yu and P. F. Bernath, , J. Mol. Spectrosc. 240, 238-243 (2006).

[13] J.-G. Wang, M. J. Dick, P. M. Sheridan, S. Yu and P. F. Bernath, J. Mol. Spectrosc. 245, 26-33 (2007).

[14] J. D. Tandy, J.-G. Wang, P. F. Bernath, J. Mol. Spectrosc. 255, 63-67 (2009).

[15] W.L. Barclay, Jr., M.A. Anderson, L.M. Ziurys, Chem. Phys. Lett. 196 (1992) 225.

[16] L.M. Ziurys, W.L. Barclay, Jr., M.A. Anderson, Astrophys. J. 384 (1992) L63.

[17] M.A. Anderson, W.L. Barclay, Jr., L.M. Ziurys, Chem. Phys. Lett. 196 (1992) 166. 
[18] M.A. Anderson, M.D. Allen, W.L. Barclay, Jr., L.M. Ziurys, Chem. Phys. Lett. 205 (1993) 415.

[19] A.J. Apponi, W.L. Barclay, Jr., L.M. Ziurys, Astrophys. J. 414 (1993) L129.

[20] N.M. Lakin, T.D. Varberg, J.M. Brown, J. Mol. Spectrosc. 183 (1997) 34.

[21] A.J. Apponi, M.A. Anderson, L.M. Ziurys, J. Chem. Phys. 111 (1999) 10919.

[22] C.J. Whitham, H. Ozeki, S. Saito, J. Chem. Phys. 110 (1999) 11109.

[23] C.J. Whitham, H. Ozeki, S. Saito, J. Chem. Phys. 112 (2000) 641.

[24] L.N. Zack, M. Sun, M.P. Bucchino, D.J. Clouthier, L.M. Ziurys, J. Phys. Chem. A 116 (2012) 1542.

[25] A.G. Adam, K. Athanassenas, D.A. Gillett, C.T. Kingston, A.J. Merer, J.R.D. Peers, S.J. Rixon, J. Mol. Spectrosc. 196 (1999) 45.

[26] Morrison, R. T., \& Boyd, R. N. 1992, Organic Chemistry (6th ed.; Edgewood Cliffs, NJ: Prentice Hall)

[27] L. Zhang, L. Shao, M. Zhou, Chem. Phys. 272 (2001) 27.

[28] M. Sun, A.J. Apponi, L.M. Ziurys, J. Chem. Phys. 130 (2009) 034309.

[29] D.T. Halfen, J. Min, A.J. Apponi, L.M. Ziurys, in prep.

[30] M. Sun, D.T. Halfen, J. Min, B. Harris, D.J. Clouthier, L.M. Ziurys, J. Chem. Phys. 133 (2010) 174301.

[31] Z. Kisiel, J. Mol. Spectrosc. 218, (2003) 58.

[32] J. Hoeft, T. Törring, Chem. Phys. Lett. 215 (1993) 367.

[33] M. Trachtman, G.D. Markham, J.P. Glusker, P. George, C.W. Bock, Inorg. Chem. 40 (2001) 4230 . 
Table 1

Transition Frequencies of YOH and $\operatorname{YOD}\left(\tilde{X}^{1} \Sigma^{+}\right)^{\mathrm{a}}$

\begin{tabular}{|c|c|c|c|c|c|c|}
\hline \multirow[b]{2}{*}{$J^{\prime}$} & \multirow[b]{2}{*}{$\rightarrow$} & \multirow[b]{2}{*}{$J^{\prime \prime}$} & \multicolumn{2}{|c|}{$\mathrm{YOH}$} & \multicolumn{2}{|c|}{ YOD } \\
\hline & & & $v_{\text {obs }}$ & $v_{o}-v_{c}$ & $v_{\text {obs }}$ & $v_{0}-v_{c}$ \\
\hline 1 & $\rightarrow$ & 0 & 17401.995 & $<0.001$ & 15657.246 & $<0.001$ \\
\hline 2 & $\rightarrow$ & 1 & 34803.838 & $<0.001$ & 31314.399 & $<0.001$ \\
\hline 3 & $\rightarrow$ & 2 & 52205.375 & $<0.001$ & & \\
\hline
\end{tabular}

${ }^{\mathrm{a}}$ In $\mathrm{MHz}$. 


\section{Table 2}

Spectroscopic Constants of YOH and YOD $\left(\tilde{X}^{1} \Sigma^{+}\right)$

\begin{tabular}{ccccc}
\hline \hline & \multicolumn{2}{c}{ YOH } & \multicolumn{2}{c}{ YOD } \\
\cline { 2 - 5 } Parameter $^{\mathrm{a}}$ & This work & Previous work $^{\mathrm{b}}$ & This work & Previous work $^{\mathrm{b}}$ \\
\hline$B$ & $8701.0103(54)$ & $8701.81(69)$ & $7828.631(10)$ & $7829.6(1.2)$ \\
$D$ & $0.00636(36)$ & $0.0064(13)$ & $0.0039(14)$ & $0.0082(17)$ \\
$r m s$ & $<0.001$ & & $<0.001$ & \\
\hline
\end{tabular}

${ }^{\mathrm{a}}$ In $\mathrm{MHz}$; errors are $3 \sigma$ in the last quoted decimal places.

${ }^{\mathrm{b}}$ Ref. [25]. 
Table 3

Structural Parameters of $\mathrm{YOH}$ and Related Molecules

\begin{tabular}{cllccc}
\hline \hline Molecule & $r(\mathrm{M}-\mathrm{O})(\AA)$ & $r(\mathrm{O}-\mathrm{H})(\AA)$ & $\begin{array}{c}\theta(\mathrm{C}-\mathrm{M}-\mathrm{C}) \\
(\mathrm{deg} .)\end{array}$ & Method & Ref. \\
\hline $\mathrm{YOH}$ & $1.949(1)$ & $0.921(1)$ & 180 & $r_{0}, \mathrm{MW}$ & This work \\
& $1.9486(4)$ & $0.921(3)$ & 180 & $r_{0}, \mathrm{LIF}$ & {$[25]$} \\
& 1.976 & 0.956 & 180 & $r_{e}, \mathrm{~B} 3 \mathrm{LYP}$ & {$[27]$} \\
$\mathrm{YO}$ & $1.790(1)$ & & & $r_{0}$ & {$[32]$} \\
$\mathrm{RbOH}$ & 2.316 & 0.913 & 180 & $r_{0}$ & {$[9]$} \\
$\mathrm{SrOH}$ & 2.111 & 0.922 & 180 & $r_{0}$ & {$[17]$} \\
$\mathrm{MgOH}$ & 1.780 & 0.871 & 180 & $r_{0}$ & {$[21]$} \\
$\mathrm{CuOH}$ & $1.77182(3)$ & $0.9646(3)$ & $110.12(30)$ & $r_{z}$ & {$[23]$} \\
$\mathrm{ZnOH}$ & $1.795(1)$ & $0.967(3)$ & $114.2(2)$ & $r_{\mathrm{m}}^{(1)}$ & {$[24]$} \\
$\mathrm{AgOH}$ & $2.01849(4)$ & $0.9639(1)$ & $107.81(2)$ & $r_{z}$ & {$[23]$} \\
\hline
\end{tabular}




\section{Figure Captions}

FIGURE 1. - Fourier transform microwave/millimeter-wave (FTMmmW) spectra of the $J=1 \rightarrow 0$

(upper panel), $2 \rightarrow 1$ (middle panel), and $3 \rightarrow 2$ (lower panel) rotational transitions of YOH

$\left(\tilde{X}^{1} \Sigma^{+}\right)$near $17.4,34.8$, and 52.2 GHz, respectively. Doppler doublets, indicated by brackets, are shown on each line profile. The spectra were each created from $600 \mathrm{kHz}$-wide scans, with 500 , 500, and 25,000 pulse averages per scan, respectively.

FIGURE 2. - FTMmmW spectra of the $J=1 \rightarrow 0$ (upper panel) and $2 \rightarrow 1$ (lower panel), rotational transitions of YOD $\left(\tilde{X}^{1} \Sigma^{+}\right)$near 15.6 and $31.3 \mathrm{GHz}$, respectively. Doppler doublets are indicated for each transition by brackets. The spectra was created from scans with 5000 pulse averages, each $600 \mathrm{kHz}$ in width. 
Figure 1

\section{YOH $\left(\widetilde{\mathrm{X}}^{1} \Sigma^{+}\right)$}
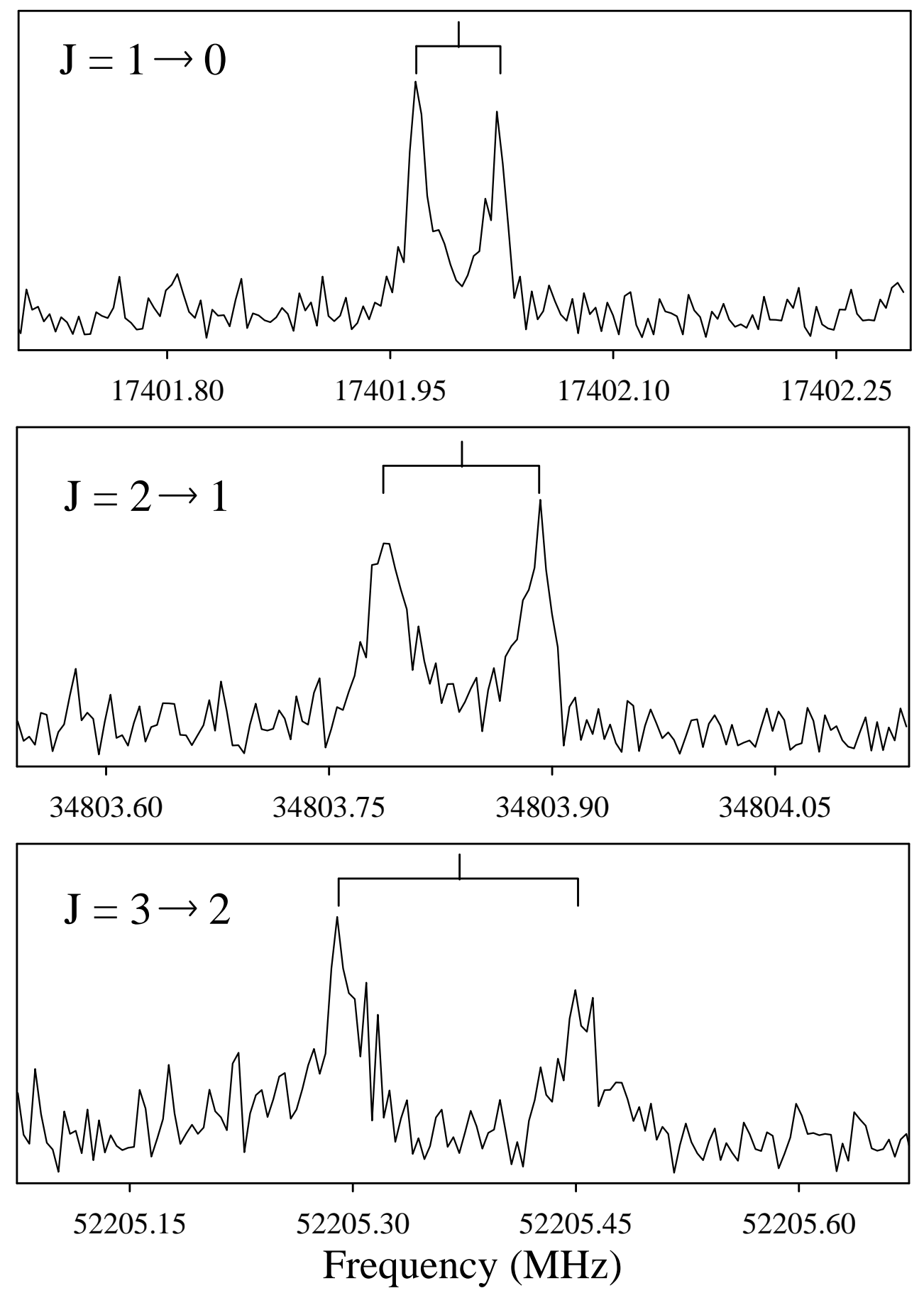
Figure 2

$\operatorname{YOD}\left(\widetilde{\mathrm{X}}^{1} \Sigma^{+}\right)$
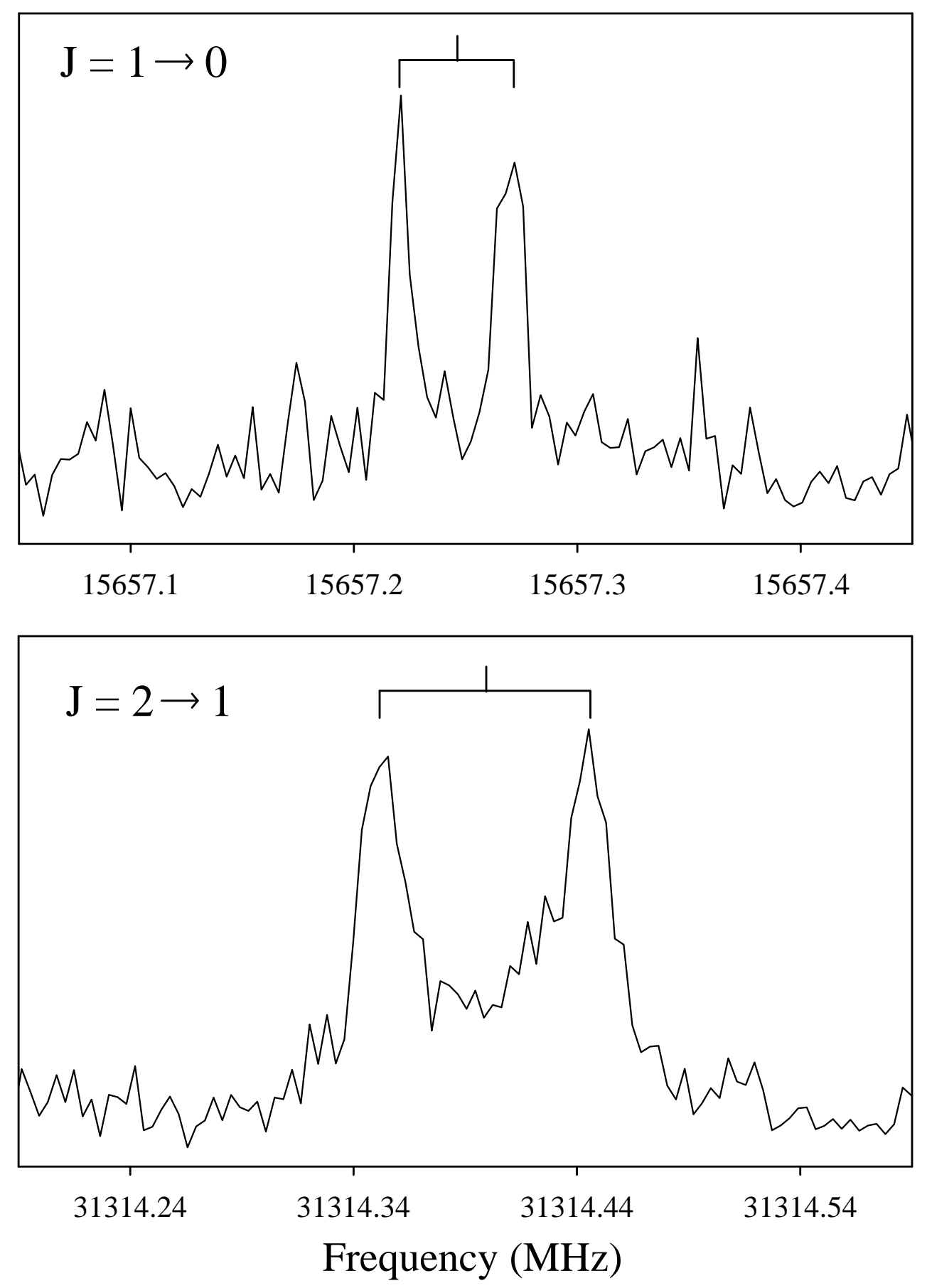


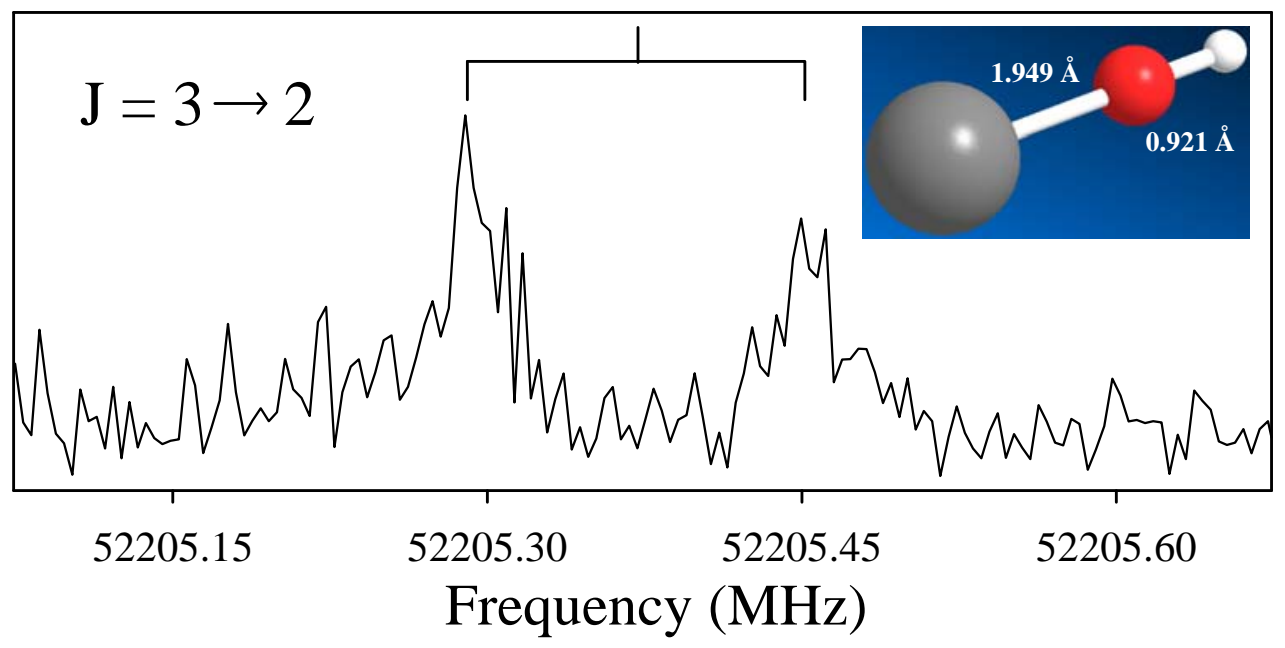

